\section{Insomnie bei Hypnotikaabhängigkeit}

Helga Peter

Marburg, Deutschland

\section{Synonyme}

Hypnotikainduzierte Insomnie

\section{Englischer Begriff}

hypnotic dependent sleep disorder

\section{Definition}

Es handelt sich um einen mittlerweile historischen Begriff für Schlafstörungen in Form von Ein- und Durchschlafstörungen sowie exzessiver Tagesschläfrigkeit, die auf Toleranzentwicklungen bei Hypnotikaeinnahme oder den Entzug von >Hypnotika“ zurückgeführt werden können.

In der ICSD-R (International Classification of Sleep Disorders - Revised) von 1997 wurde die Insomnie bei Hypnotikaabhängigkeit als extrinsische Dyssomnie gelistet, eine Kategorie, die in der ICSD-2 von 2005 aufgegeben wurde. Dort wurde sie unter der Hauptkategorie Insomnien als Insomnie durch Medikamente oder Substanzen klassifiziert. Nach > „ICSD-3“ (2014) kann eine derartige Störung als Andere Insomnie diagnostiziert werden.

Siehe auch $>$,Diagnostische Klassifikationssysteme“. 